\title{
A Two-Dimensional Brain-Computer Interface Associated With Human Natural Motor Control
}

\author{
Dandan Huang ${ }^{1}$, Xuedong Chen ${ }^{2}$, Ding-Yu Fei ${ }^{1}$ and Ou Bai ${ }^{1}$ \\ ${ }^{1}$ EEG \& BCI Laboratory, Virginia Commonwealth University \\ 2State Key Laboratory of Digital Manufacturing Equipment and Technology, \\ Huazhong University of Science and Technology \\ 1 USA \\ 2P. R. China
}

\section{Introduction}

\subsection{Target groups of brain-computer interfaces (BCls)}

Amyotrophic lateral sclerosis (ALS) is a progressive neurodegenerative disease that affects nerve cells which are responsible for controlling voluntary movement. Primary lateral sclerosis (PLS) is a variant of ALS that affects the corticospinal upper motor neurons, limiting movement. ALS/PLS patients, as well as patients disabled from other degenerative diseases or brain injuries, have difficulty with everyday motor behaviors such as moving, swallowing, and speaking. In the later stages of disease, some patients may completely lose motor function and become totally 'locked-in' (Hayashi and Oppenheimer, 2003). Loss of motor function significantly affects patients' quality of life (QoL) (Mockford et al., 2006; Bromberg, 2008; Williams et al., 2008; Lule et al., 2009) and increases the financial burden for the cost of care (Mutsaarts et al., 2004). One important component of quality of life being addressed repeatedly by patients, specifically as the disease progresses, is the ability to communicate. A braincomputer interface (BCI) or brain-machine interface (BMI), has been proposed as an alternative communication pathway, bypassing the normal cortical-muscular pathway (Joseph, 1985; Kennedy et al., 2000). BCI is a system that provides a neural interface to substitute for the loss of normal neuromuscular outputs by enabling individuals to interact with their environment through brain signals rather than muscles (Wolpaw et al., 2002; Daly and Wolpaw, 2008). Recent years have featured a rapid growth of BCI research and development owing to increased societal interest and appreciation of the serious needs and impressive potential of patients with severe motor disabilities (Birbaumer and Cohen, 2007; Daly and Wolpaw, 2008). The majority of BCI-related publications have studied performance in healthy volunteers and focused on the development of signal processing/computational algorithms to improve BCI performance (Bashashati et al., 2007). Practical BCI clinical applications for the potential patient users, however, are still limited (Birbaumer, 2006a).

\subsection{Worldwide research on Electroencephalography (EEG)-based $\mathrm{BCI}$}

The BCIs using invasive signal methods to record intracortical neuronal activities have shown great promise in direct brain control of external devices in primates, for example, to restore self-feeding by controlling a 3-D robotic arm (Velliste et al., 2008). However, due to 
the technical concerns such as associated surgical risks as well as unclear long-term benefit and robustness, non-invasive signal methods, mainly EEG, have been extensively explored because of its lower clinical threshold as well as the ease of use. Although EEG mainly supports one dimensional control (Krusienski et al., 2007; McFarland and Wolpaw, 2003), successful two-dimensional BCI has been achieved. Wolpaw's group used two channels of bipolar EEG from the two hemispheres to provide vertical and horizontal cursor control (Wolpaw and McFarland, 2004). In contrast to invasive methods, non-invasive methods feature an extremely low signal-to-noise $(\mathrm{s} / \mathrm{n})$ ratio, which is a major challenge in EEGbased BCI development. Conventionally, $\mathrm{s} / \mathrm{n}$ ratio can be improved by repeated averaging, for example, as in event-related potentials (ERPs), which can be obtained by averaging across trials time-locked to the stimuli. However, due to the requirement for repeated measurements, the communication speed is greatly reduced. An alternative method to improve $\mathrm{s} / \mathrm{n}$ ratio for reliable $\mathrm{BCI}$ control is to train users to regulate their brain activity, such as by modulation of the slow-cortical potentials (SCP) (Birbaumer et al., 2000) or the 8$12 \mathrm{~Hz}$ sensorimotor $\mathrm{Mu}$ rhythm (Wolpaw and McFarland, 1994). Once people learn to effectively regulate their brain activity, reduction of the variance in the EEG signal can be expected and as a result, the $\mathrm{s} / \mathrm{n}$ ratio is increased. However, due to the variance of spontaneous activity in EEG, long-term training is usually required for users to achieve effective and accurate regulation of either SCPs or sensorimotor Mu rhythms. The long-term training may require a couple of months to 1 or 2 years (Wolpaw and McFarland, 2004; Iversen et al., 2008b). Moreover, users may be easily fatigued from the sustained attention that is required to regulate their brain activities and as a result, render the BCI control unreliable.

\subsection{What challenges practical applications of EEG-BCI?}

Fatigue becomes serious in severely paralyzed patients who demonstrate not only reduced physical but also mental endurance (Sykacek et al., 2003; Birbaumer, 2006b). Recent pilot studies of BCI feasibility for ALS patients shows that they may not be able to learn the skills for effective regulation of brain activities because they are too weak to tolerate long-term training and/or active regulation with focused attention (Kubler et al., 1999, 2001; Hill et al., 2006). Though healthy persons or less severely paralyzed patients may operate current EEGbased BCIs efficiently (Birch et al., 2002; Blankertz et al., 2007), the performance of current BCIs in severely paralyzed patients with degenerative diseases such as ALS, however, was much lower because they were easily fatigued or could not tolerate long-term training. The accuracy was just over the random level for ALS patients, in contrast to the $90 \%$ accuracy level achieved in healthy subjects (Sellers and Donchin, 2006; Iversen et al., 2008a). Therefore, the inconvenience in operation may prevent current BCIs from practical clinical applications for severely paralyzed patients who are the users most in need of direct brain control of external devices to restore function.

\subsection{Sensorimotor Rhythm-based 2D cursor control in EEG\&BCI Lab VCU}

Sensorimotor rhythms (SMR) decrease (event-related desynchronization or ERD) with movement or preparation for movement and increase (event-related synchronization or ERS) in the post-movement period or during relaxation, based on which our 2D BCI strategy was established. We have identified that the human volition to move or cease to move associated with natural motor behavior can be reliably decoded online from EEG signals, 
where users do not need to learn vast training to regulate brain activities. We found that the discrimination of ERD from ERS was much more reliable than the discrimination of ERD from background activities in conventional BCI methods (Bai et al., 2008; Kayagil et al., 2009). A short-lasting burst of EEG oscillation, termed as beta rebound or beta-ERS, has been observed in beta band $(16-30 \mathrm{~Hz})$ over human sensorimotor area after subjects produce a self-paced movement (Salmelin et al., 1995; Pfurtscheller and Lopes da Silva, 1999; Neuper and Pfurtscheller, 2001). Though the beta rebound has been postulated as the result of afferent input (Cassim et al., 2001), other studies show that the beta rebound does not necessarily depend on motor cortex output and muscle activation, and it may reflect a shortlasting state of deactivation or inhibition of the motor cortex (Pfurtscheller, 1992; Pfurtscheller et al., 1996). The feasibility of the beta rebound for BCI application derives from the fact that beta rebound may not only occur with real physical movement but also presents with motor imagery (Pfurtscheller et al., 2005). This comes into consideration since the patients who lose their voluntary muscle contraction may only imagine movement instead of producing real movement (Bai et al., 2008). The beta rebound results in a strong synchronization, i.e. higher amplitude of rhythmic activities in beta band than background activities. As ERD features lower amplitude beta band activities, the discrimination of beta rebound or beta-ERS from beta-ERD is presumably more accurate than the discrimination of ERD from background activity. Furthermore, the beta rebound also features strict somatotopic organization (Salmelin et al., 1995), allowing for potential discrimination of different limb movements spatially according to human somatotopy. In 2008, our group implemented a synchronous sequencial binary controls approach to decode EEGs to provide 2D control of a cursor on a computer screen, with simple threshold-based binary classification of band power readings taken over pre-defined time windows during subject right hand movement/motor imagery (Bai et al., 2008). The following study, using spatial feature of the beta rebound, supports a multi-dimensional BCI by reliable decoding of intentions to move individual limbs (Huang et al., 2009). The beta-ERD and beta-ERS features associated with human natural motor control has also been further tested on six ALS or PLS patients in sequential binary control for 2D cursor control, and two patients further participated in direct two-dimensional cursor control in a single visit (Bai et al., 2010).

\section{Physiological rationale for the proposed two-dimensional $\mathrm{BCl}$}

Human somatotopic organization indicates that human limbs are controlled by contralateral brain hemispheres. Many neurophysiological and neuroimaging studies have confirmed the nature of contralateral control (Bai et al., 2005; Rao et al., 1993; Stancak and Pfurtscheller, 1996). Therefore, reliably decoding the movement intention of right and left hand, which are associated with different spatiotemporal patterns of event-related desynchronization (ERD), i.e. oscillation amplitude attenuation, and event-related synchronization (ERS), i.e. oscillation amplitude increase, may provide additional degrees-of-freedom for control. During physical and motor imagery of right and left hand movements, beta band brain activation $(15-30 \mathrm{~Hz})$ ERD occurs predominantly over the contralateral left and right motor areas. The brain activity associated with ceasing to move, the post movement ERS, can also be found over the contralateral motor areas. It suggests that the brain activity associated with four natural motor behaviors (thus, not requiring extensive training) may potentially provide four reliable features for a discrete two-dimensional control, e.g. left-hand ERD to 
command move to the left, left-hand ERS to command move up, right-hand ERD to command move to the right, and right-hand ERS to command move down. As the spatial distribution of post movement beta rebound (ERS) is more focal than ERD distribution, the detection of ERS might be potentially more reliable than ERD detection only (Pfurtscheller and Solis-Escalante, 2009). As a result, the proposed method to discriminate spatial distribution of ERD and ERS might provide more accurate classification than previous methods relying on the detection of ERD only (Neuper et al., 2005; Naeem et al., 2006). Evidence has demonstrated separate spatial patterns of ERD and ERS with physical movement, it is also important to know about the hemispheric patterns during motor imagery of limb movement which is essential for achieving purely mental control without involvement of muscle activity.

\section{Experimental paradigms}

\subsection{Data acquisition and online processing system}

We used the typical BCI system setting (Fig. 1). Participants were presented with stimuli and required to perform specific mental tasks while the electrical activity of the brains was being recorded by EEG. Relevant EEG features were extracted and then fed back to the user by so-called closed-loop BCI.

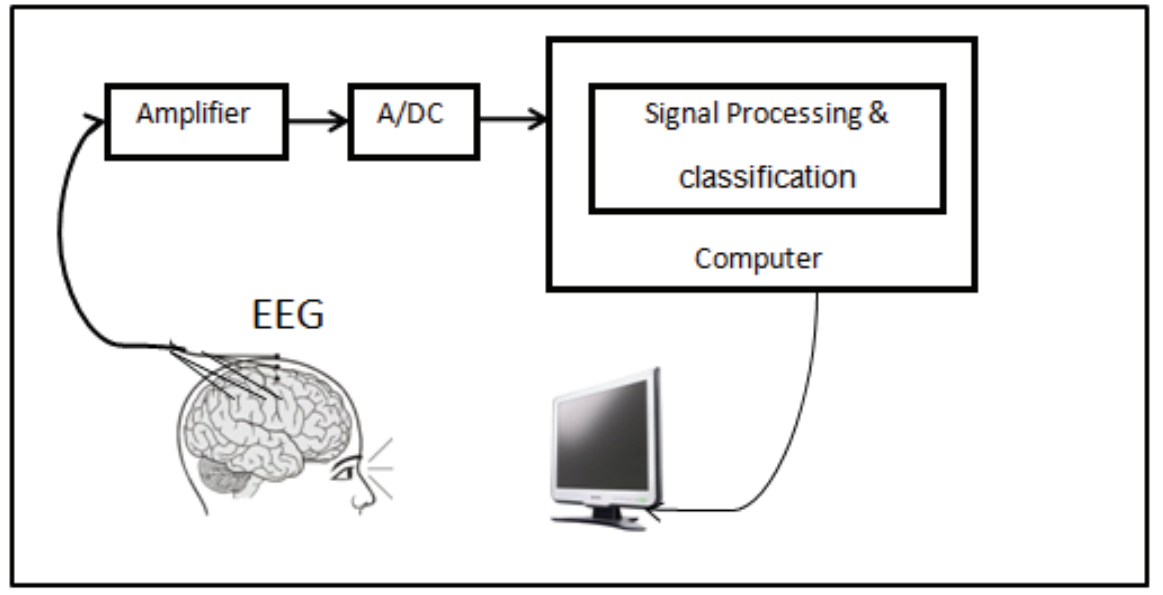

Fig. 1. Experimental system. EEG signals were picked up from scalp and amplified, then were digitized through $\mathrm{A} / \mathrm{D}$ convertor and sent to the computer for signal processing.

We recorded EEG signals from 27 (tin) surface electrodes (Fig. 2) attached on an elastic cap (Electro-Cap International, Inc., Eaton, OH, U.S.A.) according to the international 10-20 system (Jasper and Andrews, 1938), with reference from the right ear lobe and ground from the forehead. Surface electromyography (EMG), which was used to monitor the movement and bipolar electrooculogram (EOG) above left eye and below right eye were also recorded. The analog signals were amplified, and then digitized through A/D convertor. The digital signal was then sent to a computer for online processing. Signals from all the channels were amplified using a 64 channel g.USBamp-System (g.tec GmgH, Schiedlberg, Austria), filtered (0.1-100 Hz) and digitized (sampling frequency was $250 \mathrm{~Hz}$ ). 


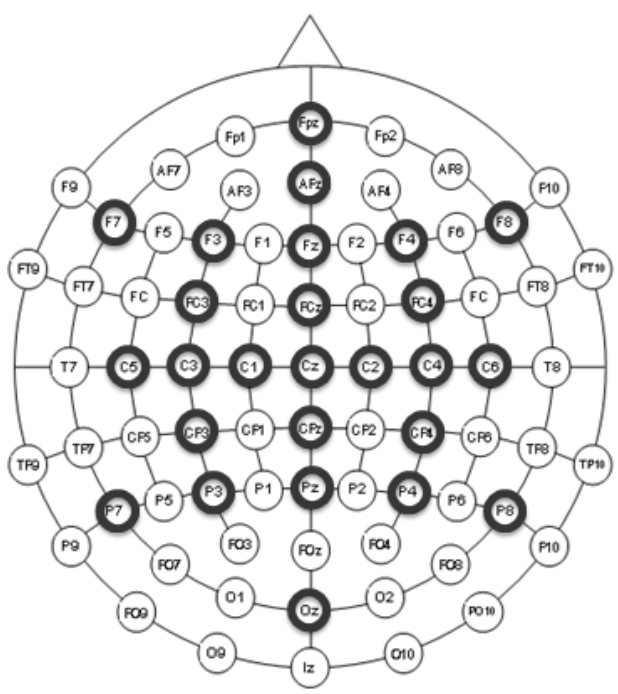

Fig. 2. Placement of 27 electrodes on the cap, marked by solid bold circles. They were F3, F7, PC3 (C3A), C1, C3, C5, T3, CP3 (C3P), P3, P7 (T5), F4, F8, PC4 (C4A), C2, C4, C6, T4, CP4 (C4P), P4, T6, FPZ, FZ, PCZ (FCZ), CZ, CPZ (CZP), PZ, OZ and AFZ was used as the ground.

\subsection{Binary and four-directional control paradigms for 2D control}

A text box was provided in the center of the computer monitor. The text message was either a blue 'Yes' or 'No' (the first cue) as illustrated in Fig. 3. Subjects were instructed to start a motor task with motor execution or motor imagery of repetitive wrist extension when they perceived the blue text message of either 'Yes' or 'No'. Subjects kept performing the motor task in the Condition window of $2.5 \mathrm{~s}$ until the color of the text message changed from blue to green (the second cue). In the 'Yes' case, subjects were instructed to continue the motor task of either motor execution or motor imagery until the text message disappeared. In the 'No' case, subjects were asked to stop the motor task and relax as soon as possible. The duration of the Detection window from text color change to text removal was also $2.5 \mathrm{~s}$. Because of the response delay, the signal from $1 \mathrm{~s}$ after color change to the end of the Detection window was extracted for classification. After an inter-trial interval randomly from 4 to $6 \mathrm{~s}$, the next text message was provided. The detailed paradigm was provided in a previous study (Bai et al., 2008; Kayagil et al., 2009). Patients participated in both motor execution and motor imagery sessions. The purpose of the motor execution session was 2fold: the patients are more comfortable with the paradigms, and the investigators could check whether patients performed the instructions properly by monitoring their motor output from EMG. One important factor was that patients need to relax as soon as possible at the beginning of the 'Detection' window in order to induce a transient feature of ERS for $\mathrm{BCI}$ detection. ERD was expected when subjects performed the active motor task during the Detection window, whereas ERS was expected when subjects stopped the motor task in the Detection window. This paradigm would yield a more accurate classification between ERD and ERS compared with that between ERD and baseline activity. 


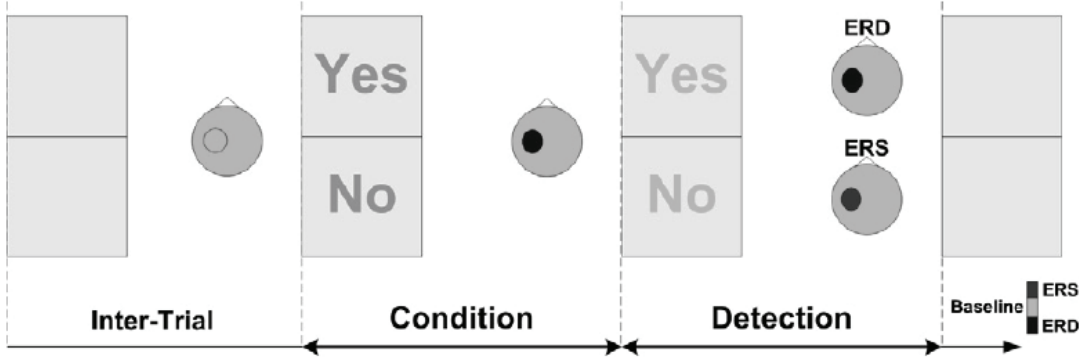

Fig. 3. Binary control paradigm. Subject started motor tasks of motor execution or motor imagery when they perceived the first color text message of either 'Yes' or 'No'. When the color of text message changed from blue to green (less dark in grey scale), subjects sustained the motor tasks in case of 'Yes' or ceased the motor tasks and relaxed in case of 'No'. EEG signal in the Detection window was extracted to determine 'Yes' from ERD activity or 'No' from ERS activity. Therefore, subjects was able to make binary control of either 'Yes' or ' $\mathrm{No}^{\prime}$ intentionally by sustaining or ceasing motor tasks time-locked to the cues (see Bai et al., 2010).

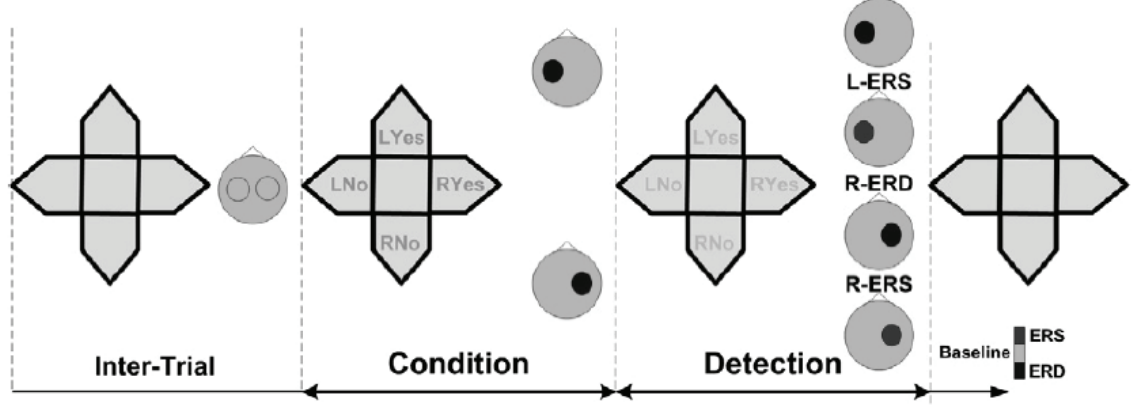

Fig. 4. Four-directional cursor control paradigm. Subjects started motor execution or motor imagery of right hand movement upon perceiving the blue text message (in Condition window) of 'RYes' or 'RNo', or left hand movement when perceiving 'LYes' or 'LNo'. They would continue the movement after text color change in cases of 'RYes' or 'LYes', or stop moving and relax in cases of 'RNo' or 'LNo'. Computer extracted EEG signal in the Detection window and decoded 'RYes', 'RNo', 'LYes' and 'LNo' from ERD and ERS over the left hemisphere, or ERD and ERS over the right hemisphere correspondingly (see Bai et al., 2010).

Each of the four text messages 'RYes', 'RNo', 'LYes' and 'LNo' was assigned to one of the four directions of a computer cursor, provided in the center of the computer monitor (Fig. $4)$. One of four text messages in the corresponding cursor direction was provided each time. The message text was a blue color at first; in the cases of 'RYes' or 'RNo', subjects started to perform motor execution or motor imagery of their right wrist in the form of repetitive extension; and in the cases of 'LYes' or 'LNo', subjects started to perform motor execution or motor imagery of their left wrist in the form of repetitive extension. Subjects kept performing the motor task until the color change of the text message. In the Detection window after the color change, subjects were instructed to continue the motor task of right 
wrist extension or left wrist extension with text messages of 'RYes' or 'LYes', respectively. Subjects were asked to cease the motor task as soon as possible and relax when they saw the messages of 'RNo' or 'LNo'. The durations of the Condition and Detection window were both $2 \mathrm{~s}$. The signal between $1 \mathrm{~s}$ after the text color change and the end of the Detection window was extracted for classification. The detailed paradigm can be found in (Huang et al., 2009). In the Detection window, the four motor tasks of 'RYes', 'RNo', 'LYes' and 'LNo' were associated with four spatial patterns of ERD over the left hemisphere, ERS over the left hemisphere, ERD over the right hemisphere and ERS over the right hemisphere according to human somatotopy of hand control. The spatial distribution of the four patterns provided the basis for the classification of 'RYes', 'RNo', 'LYes' and 'LNo' to achieve control of the four directions of the computer cursor.

\subsection{Online two-dimensional cursor control game}

A computer game of virtual computer cursor control using BCI was developed to facilitate subjects' interest and active involvement for BCI development (Kayagil et al., 2009). Subjects were asked to control the cursor movement in a two-dimensional space on the computer monitor (see Fig. 5) by performing motor tasks with either motor execution or motor imagery. The binary control of two-dimensional cursor movement was achieved by consecutive binary classification to determine one of up, down, right and left directions. Subjects were instructed to move the cursor (the dark square box) towards the target (the circle) with minimal cursor movements in the grids, and at the same time, avoid the trap (the black ghost). The initial position of the cursor as well as the target and trap position were randomly generated by the computer. Fig. 5 shows screen shots of a binary control in the up row. As the target was in the upper left direction of the cursor, the subjects would select either up or left cursor, i.e. 'No' directions. Similar to the binary control paradigm, subjects started motor task with either motor execution or motor imagery when the four text boxes were provided. Because the 'No' direction was closer to the target, subjects would stop the motor task when the cursor color changed to green so that the ERS activity was

\section{Binary Control}

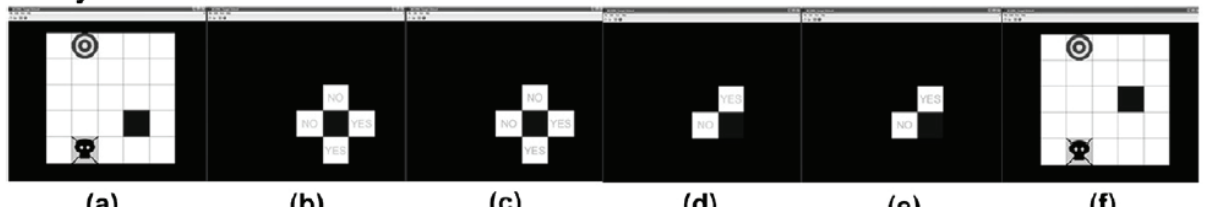

(a)

(b)

(c)

(d)

(e)

(f)

Four-Directional Control

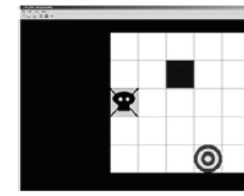

(a)

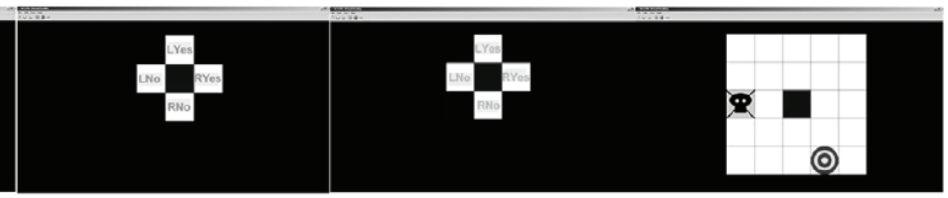

(b) (c) (d)

Fig. 5. Consecutive binary control and four-directional control of two-dimensional computer cursor movement: an online computer game to test the performance of binary control and four-directional control paradigms (see detail in the text). 
voluntarily produced. The computer determined whether the subjects intended to move to 'Yes' or 'No' direction according to the extracted EEG signal, i.e. ERS, with respect to the computer model created from the data obtained from binary control paradigm. The two 'Yes' directions were removed when the computer detected ERS signal. The two 'No' directions were changed to one 'Yes' direction and one 'No' direction, and the subjects performed the motor task to voluntarily 'tell' the computer which direction they wanted to move to. In the illustrated sample, the subjects performed a sustained movement, and the computer determined the 'Yes' direction and move the cursor upward. Similarly, subjects would control the cursor movement until it reached the target. The detailed explanation of the binary cursor control game was described in the previous study (Kayagil et al., 2009). The scheme of the four-directional control of two-dimensional cursor movement was similar to that of binary cursor control. Because one of the possible four directions was able to be determined from one of 'RYes', 'RNo', 'LYes' and 'LNo', which were provided in fourdirectional control paradigm, the consecutive two binary classification was reduced to one classification from four options as shown in the lower row in Fig.5. The detailed explanation of the four-directional cursor control game was described in (Huang et al., 2009).

\subsection{Center-out two-dimensional cursor control paradigm}

A trial began when a target (dark) appeared at one of the four locations on the periphery of the screen, together with three non-target objects on the other three sides (Fig. 6a). A target location was pseudo-randomized (i.e. each occurred the same times in one block). In both parts (physical movement and motor imagery), there were four hint words in the task paradigm (a), 'RYes', 'RNo', 'LYes', and 'LNo' ('R' indicating right hand task, and 'L' for left hand task) on the four directions of the central cursor, which was set in green initially. Subjects were instructed to begin real or imagined repetitive wrist extensions of the right arm, if the target was on the direction of 'RYes' or 'RNo'; if the target was on the direction of

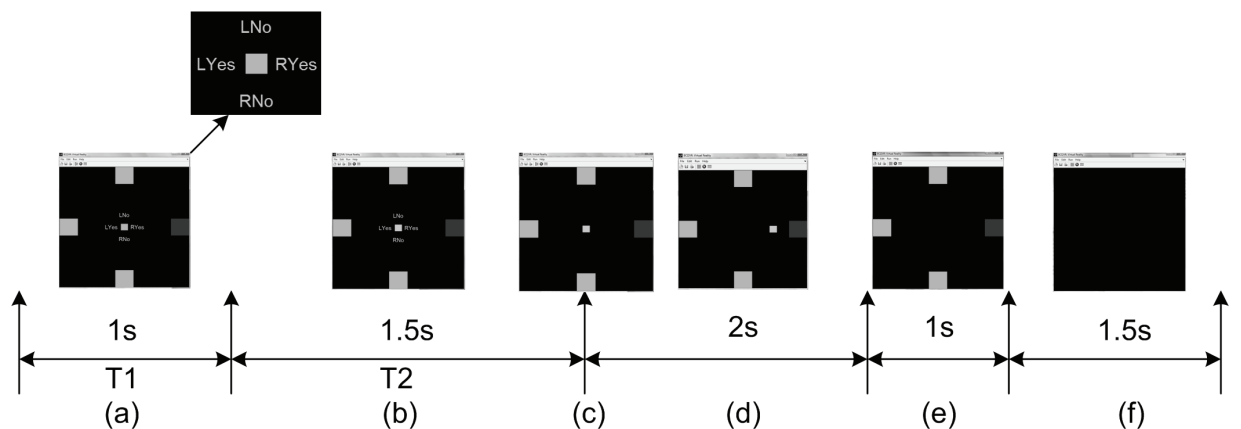

Fig. 6. Online 2D center-out cursor control paradigm. (a) A trial began. The target (dark) was pseudo-randomly chosen from the four positions along the edges; the cursor was in green. Subject started motor task for $1 \mathrm{~s}$. (b) The cursor turned to cyan, at which point subject stopped and relaxed in 'No' case, or performs sustained movement in 'Yes' case for 1.5s. (c) The hint words disappeared. Subject stopped the task. (d) The cursor moved steadily towards the classified direction for $2 \mathrm{~s}$. (e) The target flashed for $1 \mathrm{~s}$ when it was hit by the cursor. If the cursor missed the target, the screen was blank for $1 \mathrm{~s}$. (f) The screen was blank for a $1.5 \mathrm{~s}$ interval before next trail started. 
'LYes' or 'LNo', they performed real or imagined repetitive wrist extensions of the left arm. After a period of $1 \mathrm{~s}$, the central cursor changed color to blue (b), when the subject was instructed to continue real or imagined movement with the 'Yes' case or abruptly relax and stop moving with the 'No' case. After displaying for a period of $1.5 \mathrm{~s}$, the configuration disappeared, indicating that subject needed to stop the task, and the screen was blank for $4.5 \mathrm{~s}(\mathrm{f})$. Next trial began from (a).

\section{Signal processing and computational methods}

\subsection{Pre-processing}

EEG signal in the Detection window was extracted for modeling and classification. Signals from 27 channels were spatially filtered by surface Laplacian derivation (SLD), i.e. signal from each electrode was referenced to the averaged potentials from the nearby four orthogonal electrodes (Hjorth, 1975). The temporal filtering was achieved by power spectral estimation with Welch method. A $4 \mathrm{~Hz}$ frequency resolution with segment length of $0.25 \mathrm{~s}$ and $50 \%$ overlapping was determined for spectral estimation (Bai et al., 2007).

\subsection{Feature extraction}

Empirical feature reduction: assuming that movement intention associated cortical activities occur over the motor cortex, we reduced the channel number from 27 to 14 , which covered both left and right motor areas. Furthermore, as we did not expect relevant activities in the delta, theta or gamma band, only alpha and beta band $(8-30 \mathrm{~Hz})$ activities were extracted for modeling and classification. Thus, the total number of extracted features were 8 (frequency bins) ${ }^{*} 14$ (channels) $=112$ features.

Bhattacharyya distance: Bhattacharyya distance provides an index of feature separability for binary classification, which is proportional to the inter-class mean difference divided by intra-class variance (Chatterjee et al., 2007). The empirically extracted features were ranked by the Bhattacharyya distance for further classification.

Genetic algorithm: Genetic algorithm (GA)-based feature selection is a stochastic search in the feature space guided by the concept of inheriting, where at each search step, good properties of the parent subsets found in previous steps are inherited. Ten fold crossvalidation was used with a Mahalanobis Linear Distance (MLD) classifier for feature evaluation ( $\mathrm{Li}$ and Doi, 2006). In this approach, the population size we used was 20, the number of generations was 100 , the crossover probability was 0.8 , the mutation probability was 0.01 , and the stall generation was 20 .

\subsection{Classification}

ROC: A receiver operating characteristics (ROC) was generated from the feature with the largest Bhattacharyya distance, i.e. the one providing the largest inter-class separability. The working point was determined from the ROC curve that was the closet point to $100 \%$ true positive with $0 \%$ false positive.

GA-MLD: The sub-optimal feature subset was selected by genetic algorithm (GA) with Mahalanobis Linear Distance (MLD) as the evaluation function. Then, the selected features providing the best cross-validation accuracy were applied to a Mahalanobis Linear Distance Classifier. The number of features for the subset was four, which was determined from the cross-validation accuracy with feature numbers of 2, 4, 6, 8 and 10. 
SVM: the support vector machine (SVM) was employed as the evaluation function. We employed a SVM approach provided in LIBSVM (Fan et al., 2005). The radial basis function was used as the SVM kernel function as it can provide similar classification outcome compared with other kernels (Keerthi and Lin, 2003). As the performance of SVM depends on the regulation parameters or hyper-parameters $C$ and the width of the kernel $r$ (Chang and Lin, 2001; Muller et al., 2001), 10-fold cross-validation was performed; $2^{\mathrm{K}}, \mathrm{K}$ from -5 to 15 with step of 2 for the penalty parameter and $2^{K}, K$ from -15 to 5 with step of 2 for the spread parameter. These parameters were determined by the training dataset only.

\subsection{Flow chart of online calibration and two-dimensional cursor control games}

Fig.7 illustrates the general procedures for online calibration and online 2D cursor control games. In calibration step, data was first spatially filtered using surface Laplacian derivation (SLD), and then was temporally filtered by estimation of the power spectral density. Through offline neurophysiological analysis, 0.5-1.5 s after T2 window started was selected to obtain strongest ERD/ERS. We applied Welch method with Hamming window, and kept the frequency resolution $4 \mathrm{~Hz}$, the same as previous study, with $50 \%$ overlap of the segments. For either physical movement or motor imagery, parameters and features were determined from the training data to make a model, used for decoding the movement intention in online tests. We performed empirical feature reduction by empirical feature reduction of channel and frequency band restrictions. GA-MLD and DTC were used to generate models. The online data also went through spatial filtering, temporal filtering, channels and frequency bands restriction. The cursor was moved to the classified direction. For the center-out paradigm, where we performed model adaptation, trials were combined with the old ones, keeping the data pool updated. New models would be generated using MLD and DTC, the one with higher accuracy would be used as the classifier in next trial. If the block was completed, the features were re-selected by genetic algorithm, and new models were generated by GA-MLD and DTC. Next block began with the same procedures.

\subsection{Offline cross-validation}

The offline performances were evaluated from 10 -fold cross-validation; $90 \%$ of the data pool was used for training, and the other $10 \%$ was used for validation so that the validation dataset was independent from the training dataset. In the online game, the features for decoding the movement intention was extracted and classified using the parameters determined from the training datasets.

\subsection{Data processing for neurophysiological analysis}

Offline data analysis was performed to investigate the neurophysiology following the tasks of 'Yes' and 'No' for binary classification, and 'RYes', 'RNo', 'LYes' and 'LNo' for fourdirectional classification. The calibration datasets were used for analysis. Data processing was performed using MATLAB Toolbox: BCI2VR (Bai et al., 2007). Epoching was done with windows of $-2 \mathrm{~s}$ to $7 \mathrm{~s}$ with respect to the first cue onset. Any epochs contaminated with artifacts were rejected. ERD and ERS were calculated for each case. Epochs were linearly detrended and divided into $0.25 \mathrm{~s}$ segments. The power spectrum of each segment was calculated using FFT with Hamming window resulting in a bandwidth of $4 \mathrm{~Hz}$. ERD and ERS were obtained by averaging the log power spectrum across epochs and having baseline corrected with respect to $-2 \mathrm{~s}$ to $0 \mathrm{~s}$. 


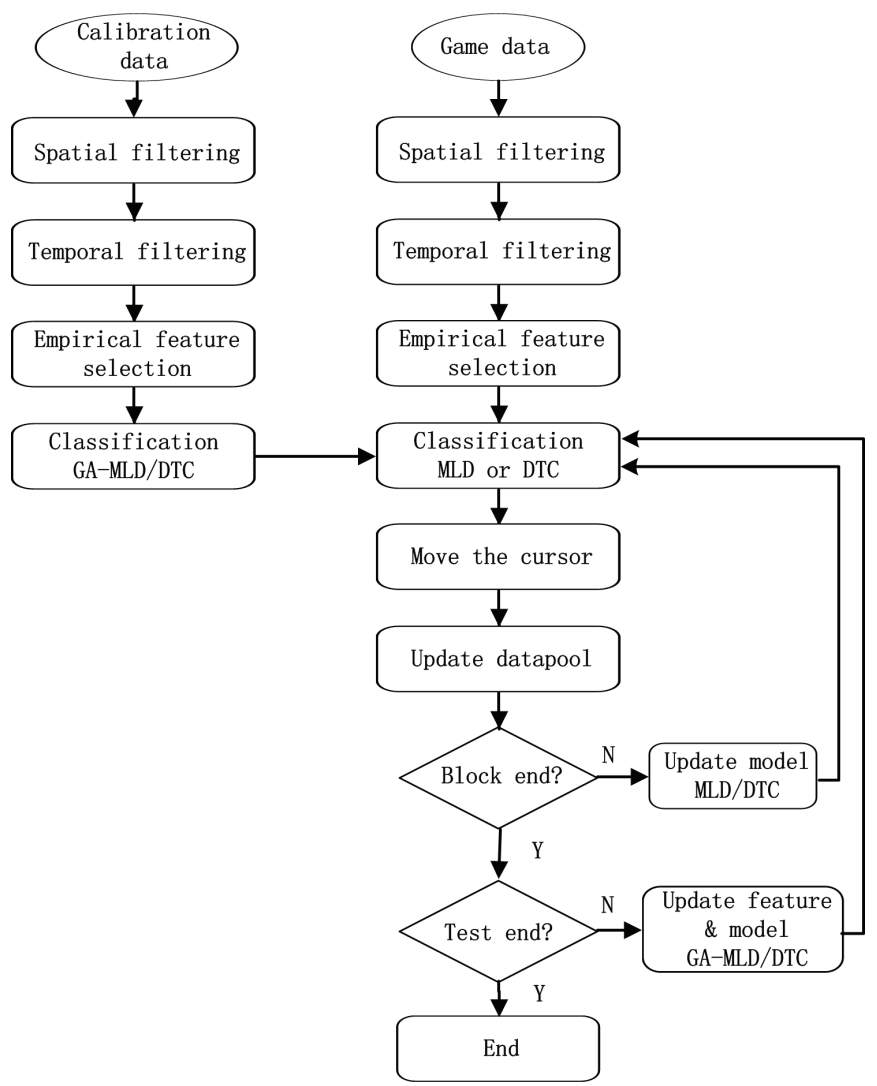

Fig. 7. Flow chart of online calibration and two-dimensional cursor control games.

Calibration data went through spatially filtering, temporally filtering and empirical feature selection. In classification, genetic-algorithm based Mahalanobis linear discrimination (MLD) classifier and decision tree classifier (DTC) were used to generate models for online game. During the online test, data was spatially filtered, temporal filtered, and empirical features were selected. Then the model generated in calibration step, giving a better prediction result was used to classify the movement intention, and the cursor was moved. For the later study with center-out 2D paradigm, after data pool was updated, the model was updated consequently, using MLD and DTC, and the one giving a higher result was used as the model for classification in next trial; if the block ended, features would be reselected by genetic algorithm. If all the blocks were completed, the procedure ended.

\section{Observations and experiment results}

\subsection{ERD/ERS in healthy subjects and in ALS/PLS patients}

For ERD/ERS patterns in ALS/PLS patients during binary control in order to realize 2-D control, results were shown in (Bai et al., 2010). We selected to present results of healthy and ALS1/PLS1 patients in four-directional control, which included patterns from all four motor tasks. 


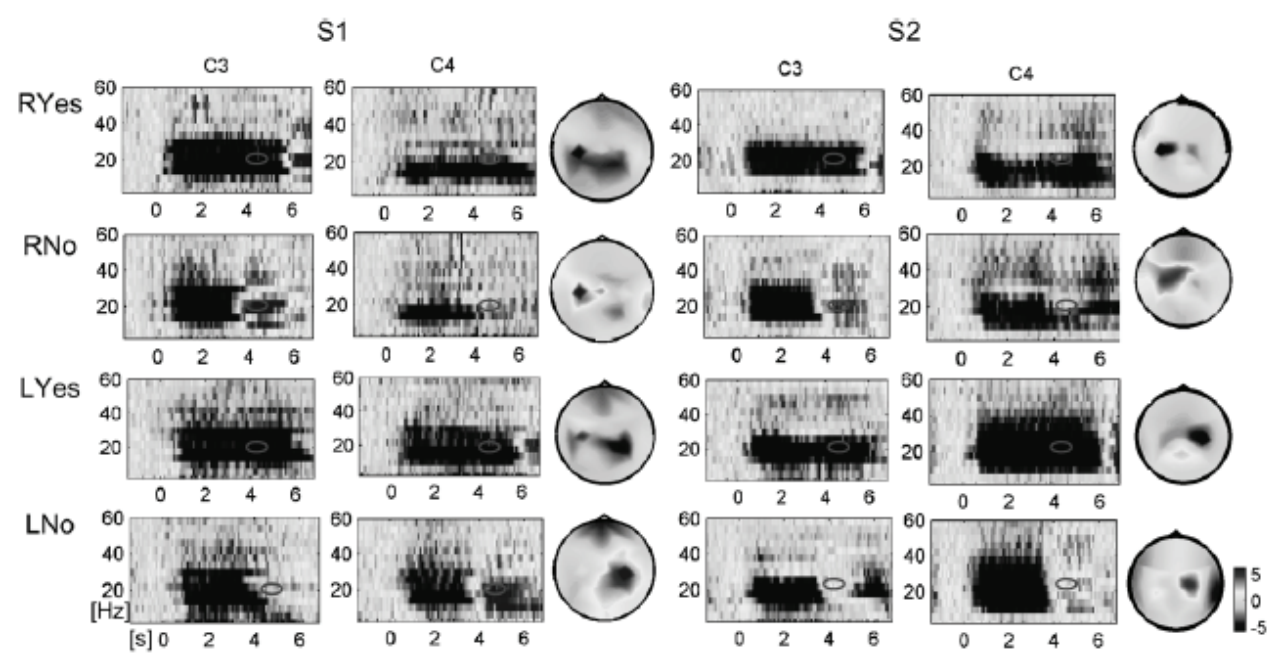

Fig. 8. Time-course and topography of ERD and ERS during motor execution following the calibration paradigm for healthy subjects S1and S2. Blue color stands for power decrease or ERD; red stands for power increase or ERS (see original color picture in Huang et al., 2009). The T1 window is from $0 \mathrm{~s}$ to $2.5 \mathrm{~s}$ and the T2 window is from $2.5 \mathrm{~s}$ to $5 \mathrm{~s}$. ERD was observed in the T2 window on the left hemisphere during sustained right-hand movement; ERS was observed in the T2 window on the left hemisphere when the subjects ceased to move righthand movement. During left-hand movement, ERD was observed in the T2 window on the right hemisphere during sustained movement and ERS on the right hemisphere when the subjects ceased to move left hand. ERD and ERS in each case were marked by pink circles in the time-course plot. The head topography corresponding to the pink marked time period was provided next to the time-course plots.

Fig.8 shows an example of time-frequency plots, head topographies of ERDs or ERSs for motor execution with physical movement, from two healthy subjects. For each subject, timefrequency plots of channel C3 over the left sensorimotor cortex and C4 over the right hemisphere are illustrated in the left two columns and head topography of ERD or ERS to their right, containing each of the four situations: 'RYes', 'RNo', 'LYes' and 'LNo'. In the time- frequency plot, $0 \mathrm{~s}$ stands for the first cue (green in the visual paradigm) occurrence. ERD (blue color) was observed from around 0.5-1 s after the cue onset due to the response delay; for S1, S2 and S3, ERD in both alpha and beta bands from 10-30 Hz was observed over motor areas contralateral to the hand moved. The ERS in red color was mainly observed in the beta band centered around $20 \mathrm{~Hz}$ over the contralateral motor areas. Compared with ERD patterns, ERS was shortlasting in time but highly distinguishable. Therefore, the ERD and ERS on either left or right hemisphere provided four spatial patterns to detect 'RYes', 'RNo', 'LYes' and 'LNo' intentions.

Fig.9 shows the time-frequency plots and head topography of ERD and ERS associated with motor imagery. Similar to the patterns associated with physical movement, ERD associated with motor imagery was observed in both alpha and beta bands on the contralateral hemisphere with the hand moved, although ERD amplitude was smaller than that of physical movement. ERS in the T2 window was observed on the contralateral hemisphere in beta band (13-24 Hz) only, and its amplitude was smaller than that of physical movement. 


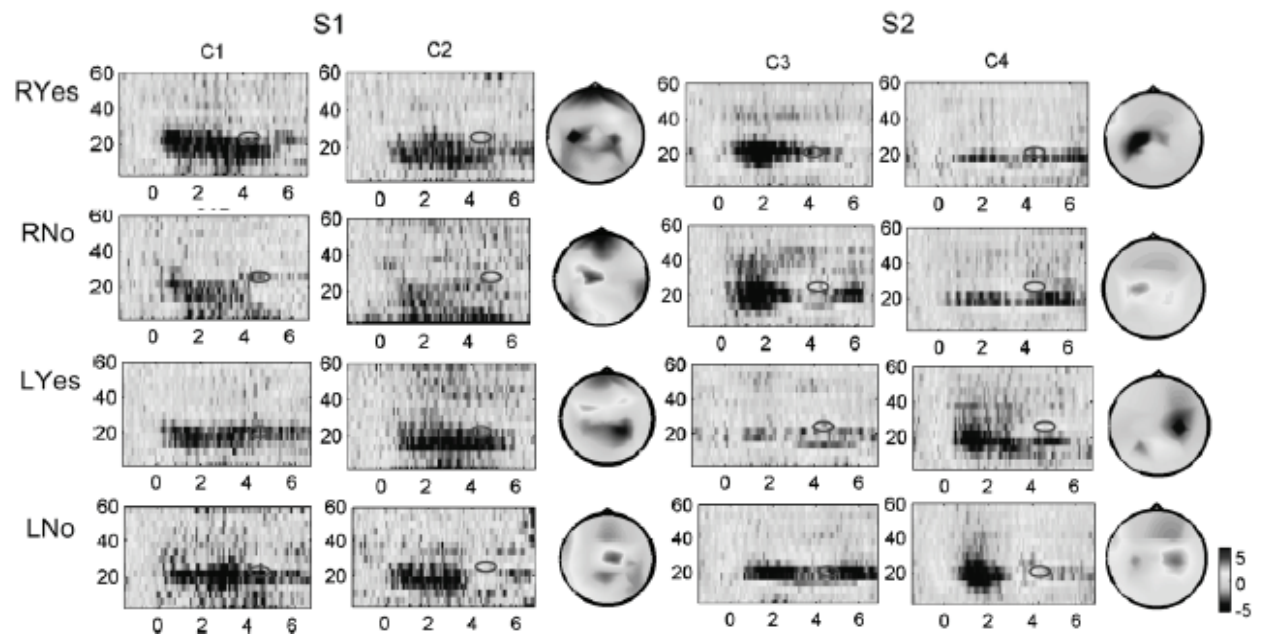

Fig. 9. Time-course and topography of ERD and ERS during motor imagery following the calibration paradigm for healthy subjects S1 and S2. For both S1 and S2, ERD is obtained in the time window with sustained motor imagery and ERS with termination of motor imagery. ERDs appear in both alpha and beta bands, bilateral, whereas ERSs appear only in the alpha band on the contralateral hemisphere. ERD and ERS in each case were marked by pink circles in the time-course plot. The head topography corresponding to the pink marked time period is provided next to the time-course plots (see original color picture in Huang et al., 2009).

During left hand motor imagery for S1 ('LYes'), ERD in the T2 time window was also observed on the left hemisphere. The ERD and ERS associated with motor imagery also provided four spatially differentiable patterns; however, the smaller amplitudes of ERD and ERS with motor imagery may result in less effective detection in single trials.

In the further study with patients, ALS1 and PLS1 participated in the additional session of four-directional control. ERD and ERS associated with motor execution were presented over left and right hemispheres corresponding to right hand and left hand movements as illustrated in Fig. 10. ERD was observed over contralateral hemispheres to the right and left hand for both subjects. Similar to the ERD pattern in binary control paradigm, ipsilateral ERD was also seen in ALS1 during the active motor task. The contralateral ERS after active motor task was clearly seen in PLS1, whereas ERS pattern was not distinguishable in ALS1. In the motor imagery experiment, ALS1 was not able to cease the motor task as soon as the color was changed. The subject reported that the muscle stiffness delayed her relaxation response. The time-frequency analysis was not presented because the ERD and ERS patterns were not distinguishable.

\subsection{Feature selection and classification}

The best frequency bands and channels for classifying movement intentions were determined from the calibration data sets. Fig.11 shows the spatial-frequency feature analysis indexed by the Bhattacharyya distance for S1 and S2 during motor execution with physical movement and motor imagery. All the channels over the whole head were used for plot. The first column for each subject illustrates the channel-frequency plot of the 

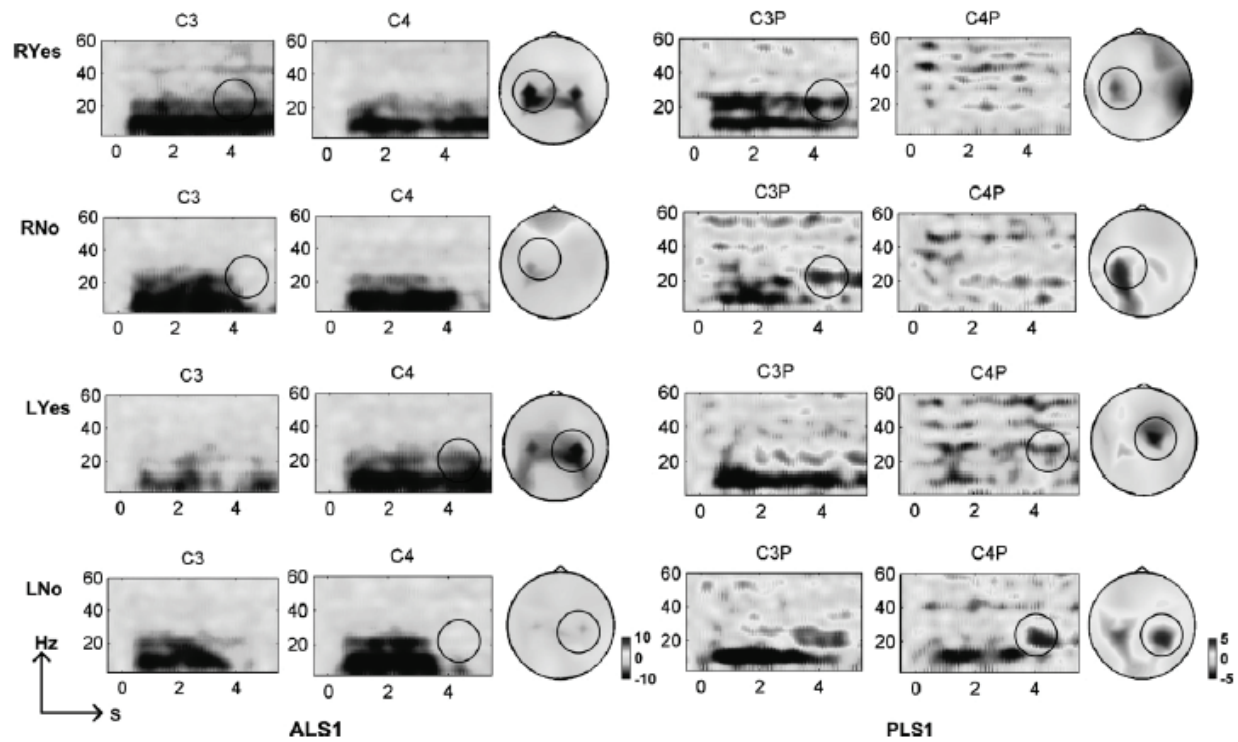

Fig. 10. Example of ERD and post-beta ERS activity over left and right motor areas associated with motor execution of right and left hand movement in the four-directional control paradigm for patients ALS1 and PLS1. ERD was detected over the motor area contralateral to the hand moved in both ALS1 and PLS1: ERD on the left hemisphere contralateral to right hand moved in case of 'RYes', and ERD on the right hemisphere contralateral to left hand moved in case of 'LYes'. Contralateral ERS to hand moved was distinguishable in PLS1: ERS on the left hemisphere contralateral to right hand moved in case of 'RNo', and ERS on the right hemisphere contralateral to left hand moved in case of 'LNo'. However, post-beta ERS in ALS1 was not recognizable (see original color picture in Bai et al., 2010).

Bhattacharyya distance, and the second column is the topography of the Bhattacharyya distance of the best frequency band. In the Bhattacharyya distance plot, the dark red color shows the higher Bhattacharyya distance standing higher separability to classify movement intentions from single trial EEG signal. In the channel-frequency plot for S1, the higher Bhattacharyya distance value for right-hand physical movement was observed in beta band ranging from 17 to $24 \mathrm{~Hz}$ in the channels located on the left hemisphere over the sensorimotor area. The high separability between ERD and ERS in the beta band was consistent with the time-frequency analysis in time-frequency plot. The topography of the Bhattacharyya distance around 17-24 Hz shows that the best EEG spatial channels for the classification of 'RYes' and 'RNo' were in the contralateral left hemisphere over the sensorimotor area since ERS presented on contralateral left hemisphere only, although ERD occurred bilaterally in time-frequency plot. A higher Bhattacharyya distance value for lefthand physical movement was also seen in the beta band on the contralateral right hemisphere. For S2, the distribution of Bhattacharyya distance values was similar to that of S1, except that for either right hand or left hand, the 'Yes' case showed high separability only on the contralateral hemisphere, which can be seen in the topography of the Bhattacharyya distance. Compared with physical movement, separability of mental tasks in 
motor imagery was weaker, indicated by lighter red area in the right two columns. Figure 7 shows feature analysis for S1 and S2 with motor imagery. The highest Bhattacharyya distance values were in the beta band and on the channels over contralateral hemisphere for both right and left-hand motor imagery.
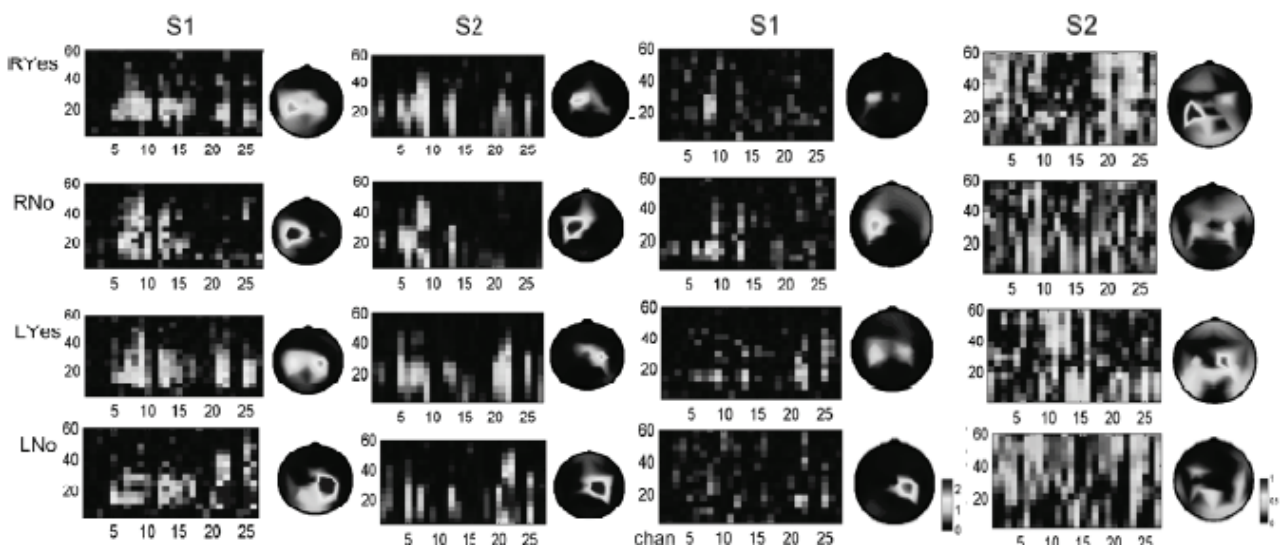

Fig. 11. Feature visualization indexed by Bhattacharyya distance for healthy subjects S1 and S2 in physical movement (left two columns) and in motor imagery (right two columns) following the calibration paradigm. The best frequency band with the highest separability was found in beta band, and the best channel was found on sensorimotor areas (see original color picture in Huang et al., 2009).
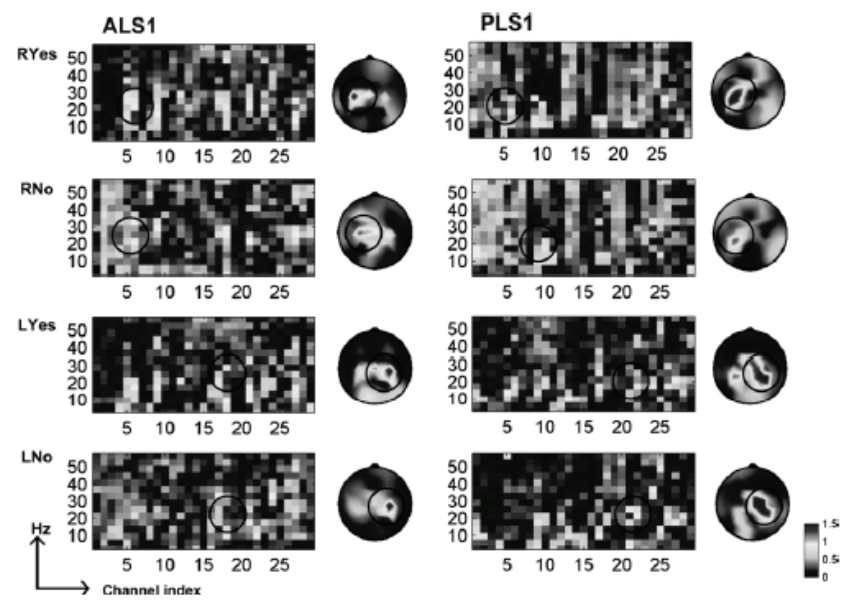

Fig. 12. Bhattacharyya distance for patient ALS1 and PLS1 in selecting better spatiotemporal features for four-directional classification. The frequency power features over left motor areas in beta band provided better detection of 'RYes' and 'RNo' associated with right hand movement, whereas the frequency power features over right motor areas in beta band provided better detection of 'LYes' and 'LNo' associated with left hand movement (see original color picture in Huang et al., 2009). 


\begin{tabular}{ccccc}
\hline Subject & \multicolumn{2}{c}{ MLD $(\%)$ GA-MLD $(\%)$} & DTC $(\%)$ & SVM $(\%)$ \\
\hline S1 & $63.1 \pm 4.51$ & $87.7 \pm 1.29$ & $87.8 \pm 1.47$ & $87.8 \pm 1.31$ \\
S2 & $79.5 \pm 6.21$ & $93.0 \pm 1.97$ & $85.5 \pm 3.87$ & $90.0 \pm 3.12$ \\
S3 & $67.3 \pm 3.04$ & $85.2 \pm 0.95$ & $84.5 \pm 2.30$ & $88.9 \pm 1.04$ \\
S5 & $71.0 \pm 2.18$ & $87.2 \pm 0.58$ & $87.7 \pm 1.75$ & $85.8 \pm 2.13$ \\
\hline Average & $70.2 \pm 6.97$ & $88.3 \pm 3.33$ & $86.4 \pm 1.64$ & $88.1 \pm 1.79$ \\
\hline
\end{tabular}

Table 1. 10-fold cross-validation accuracy. MLD, Mahalanobis linear discrimination; GAMLD, genetic algorithm-based Mahalanobis linear discrimination; DTC, decision tree classifier; SVM, support vector machine classifier.

The Bhattacharyya distance was also analyzed from the activity associated with fourdirectional control paradigm. Fig. 12 shows the Bhattacharyya distance values in ALS1 and PLS1 who performed motor execution. In consistence with the ERD and ERS patterns presented in Fig. 10, the better features to classify one of four directions from the other three directions were the activities in the beta band over the motor cortex contralateral to the hand moved. The EEG activities over central-medial area in PLS1 also provided good discrimination of 'LYes' and 'LNo'.

For study with five healthy subjects, Table 1 provides the comparison of offline 10-fold cross-validation accuracies using MLD, GA-MLD, DTC and SVM methods for S1, S2, S3 and S5 during physical movement. Since ERD and ERS patterns were not strong enough for S4, we excluded it from further exploration of classification methods. MLD has a mean value of $70.2 \%$; after applying genetic algorithm in feature selection, GA-MLD provides an improved mean value of $88.3 \%$. A paired $t$-test suggested that GA-MLD had a significant improvement of the classification accuracy over the MLD $\left(t=7.64, \mathrm{df}=3, p\right.$-value $\left.<0.01^{*}\right)$. Similarly, we also compared DTC and SVM performance with that of MLD and found that DTC and SVM outperformed MLD significantly while they two had no great difference. Since there was no significant difference among the intensive methods, the DTC method was employed for the online 2D cursor control game. Except for S4, all the other four subjects were successful in controlling the cursor moving to the target by physical movement and the average online game performances for S1, S2, S3 and S5 were $92 \%, 85 \%$, $81 \%$ and $84 \%$, with the overall performance of $85.5 \% \pm 4.65 \%$. S1 and S2 participated in the second session performing motor imagery tasks. The offline classification accuracy for S1 was $73 \% \pm 5.97 \%$, and for S2 was $59.2 \% \pm 3.63 \%$, which were lower than those of cursor control with physical movement. The two subjects reported good concentration throughout the recording, except that S2 felt sleepy in a short period in the middle. Online 2D cursor control game using motor imagery was performed by S1 and S2. S1 was able to move the cursor to the target. However, S2 performed less well than S1. The performance was consistent with the classification results for motor imagery.

To further investigate the performance of proposed 2D BCI, the four-directional classification result for ALS1 and PLS1 was provided in Table 2. The four-direction classification accuracy was about $60 \%$ for motor execution, which was much higher than the random level of $25 \%$ in the case of 4 -class discrimination. The subjects also reported that it was more difficult to imagine wrist movement of the non-dominant left hand than the dominant right hand. An appropriate training to teach effective motor imagery maybe necessary for this motor imagery task. The online game provided a better accuracy than that of offline analysis of data recorded using the four-direction control paradigm. A possible 


\begin{tabular}{|c|c|c|c|c|c|c|}
\hline & \multicolumn{3}{|c|}{ Motor Execution } & \multicolumn{3}{|c|}{ Motor Imagery } \\
\hline & \multicolumn{2}{|c|}{ Offline Cross-Validation } & \multirow{2}{*}{$\begin{array}{l}\text { Online } \\
\text { GA- } \\
\text { MLD }\end{array}$} & \multicolumn{2}{|c|}{ Offline Cross-Validation } & \multirow{2}{*}{$\begin{array}{l}\text { Online } \\
\text { Game* } \\
\text { GA- } \\
\text { MLD }\end{array}$} \\
\hline & GA-MLD & GA-SVM & & GA-MLD & $\begin{array}{l}\text { GA- } \\
\text { SVM }\end{array}$ & \\
\hline ALS1 & $52.5 \pm 6.4$ & $47.3 \pm 4.4$ & 52.0 & $42.1 \pm 4.7$ & $39.1 \pm 4.5$ & 59.7 \\
\hline PLS1 & $67.1 \pm 2.6$ & $61.5 \pm 5.6$ & 71.0 & $43.9 \pm 3.6$ & $31.0 \pm 6.9$ & 55.3 \\
\hline Average & $59.8 \pm 10.3$ & $54.4 \pm 10.0$ & 61.5 & $43.0 \pm 1.3$ & $35.1 \pm 5.7$ & 58.0 \\
\hline
\end{tabular}

Table 2. Decoding four-directional movement intention from lateral ERD and beta-ERS associated with human natural motor behavior. *Estimated from cursor trajectory towards target.

reason might be that subjects were more actively involved with the interactive game than the paradigm without performance feedback. Further, subjects might be able to adapt to the computer model for the classification from the cursor movement feedback.

\subsection{Information transfer rate of the $\mathrm{BCl}$}

The BCI performance can be evaluated from both the decoding rate and accuracy (Wolpaw et al 2002). Wolpaw et al introduced the information transfer rate (ITR) for a BCI as bits per minute (bpm) as a good measurement for both decoding rate and accuracy (Wolpaw et al 2000). In our study of $2 \mathrm{D}$ control for healthy subjects, accuracies for physical movement ranged from $85.2 \%$ to $93.0 \%$ (given by GA-MLD, although not significantly better than DTC and SVM), with the average of $88.3 \%$; for a fourclass mental task, ITR was from 1.16 bits per trial, to 1.37 bits per trial, with the average 1.29 bits per trial. For motor execution with physical movement, the total duration of T1 and T2 windows was 5 s, i.e. 12 trials per minute. Therefore, the ITR was 13.9-16.5; the average was 15.5 bits per minute. Similarly, for motor imagery, the ITR was 4.15 bits per minute to 8.03 bits per minute. The cueing period T1 is important as it left enough time for the subjects to prepare for the movement. The results were comparable in terms of both accuracy and decoding rate with previous studies (see review in Wolpaw et al (2002)). In the study where center-out paradigm was adopted, T1 window was further shortened and the subjects can still make rapid response, and as a result, the ITR was further improved. As we also consider that the control performance/accuracy is very important in practical BCI application since BCI is intended for patients having limited motor function which features extremely slowness in motor control, it may be appropriate to have limited communication speed, whereas the accuracy needs to be high enough so that the users may avoid frustration when using BCI.

\section{Conclusion}

We analyzed ERD and ERS activity from EEG associated with human natural motor control in healthy people and ALS/PLS patients. ERD associated with active motor control and post beta-ERS associated with cessation of active motor control were preserved in four out of five healthy subjects and all six ALS and PLS patients participating in this study. ERD and ERS occurred not only with motor execution with physical movement, but also with motor imagery without overt movement. The amplitudes of ERD and ERS were less with motor imagery than during motor execution. In this study, we verified that the difference between ERS and ERD 
provided better contrast than the difference between idle state or baseline activities and ERD in not only healthy subjects but also ALS and PLS patients. The better contrast provided better classification rate by reducing the inter-class pattern overlapping. Under the proposed ERD and ERS-based paradigm, subjects achieved a high accuracy of binary control $(80-90 \%$ for motor execution/motor imagery) despite not receiving extensive training. The accuracy for four-directional control was also much higher than the random level, though further training of effective motor imagery of right and left hand might be required.

The successful test on the ERD and ERS-based method associated with human natural motor control will promote the development of a practical user-friendly BCI because long-term training becomes unnecessary. This is important for severely affected patients who are unable to tolerate prolonged training. Further, users may not need to keep sustained attention to regulate EEG rhythm in the proposed $\mathrm{BCI}$ associated with human natural motor control.

\section{References}

Bai O, Lin P, Huang D, Fei DY, Floeter M.K. (2010) Towards a user-friendly brain-computer interface: initial tests in ALS and PLS patients. Clin Neurophysiol 121: 1293-303.

Bai O, Lin P, Vorbach S, Floeter MK, Hattori N, Hallett M (2008) A high performance sensorimotor beta rhythm-based brain-computer interface associated with human natural motor behavior. J Neural Eng 5: 24-35

Bai O, Lin P, Vorbach S, Li J, Furlani S, Hallett M (2007) Exploration of computational methods for classification of movement intention during human voluntary movement from single trial EEG. Clin Neurophysiol 118: 2637-2655

Bai O, Mari Z, Vorbach S and Hallett M (2005) Asymmetric spatiotemporal patterns of event-related desynchronization preceding voluntary sequential finger movements: a high-resolution EEG study Clin Neurophysiol 116 1213-21

Bashashati A, Fatourechi M, Ward RK, Birch GE (2007) A survey of signal processing algorithms in brain-computer interfaces based on electrical brain signals. J Neural Eng 4: R32-57

Birbaumer N, Kubler A, Ghanayim N, Hinterberger T, Perelmouter J, Kaiser J, Iversen I, Kotchoubey B, Neumann N, Flor H (2000) The thought translation device (TTD) for completely paralyzed patients. IEEE Trans Rehabil Eng 8: 190-193

Birbaumer N, Cohen LG (2007) Brain-computer interfaces: communication and restoration of movement in paralysis. J Physiol 579: 621-636

Birbaumer N (2006b) Breaking the silence: brain-computer interfaces (BCI) for communication and motor control. Psychophysiology 43: 517-532

Birbaumer N (2006a) Brain-computer-interface research: coming of age. Clin Neurophysiol 117: $479-483$

Birch GE, Bozorgzadeh Z, Mason SG (2002) Initial on-line evaluations of the LF-ASD braincomputer interface with able-bodied and spinal-cord subjects using imagined voluntary motor potentials. IEEE Trans Neural Syst Rehabil Eng 10: 219-224

Blankertz B, Dornhege G, Krauledat M, Muller KR, Curio G (2007) The non-invasive Berlin Brain-Computer Interface: fast acquisition of effective performance in untrained subjects. Neuroimage 37: 539-550

Bromberg MB (2008) Quality of life in amyotrophic lateral sclerosis. Phys Med Rehabil Clin N Am 19: 591-605, x-xi 
Cassim F, Monaca C, Szurhaj W, Bourriez JL, Defebvre L, Derambure P, Guieu JD (2001) Does post-movement beta synchronization reflect an idling motor cortex? Neuroreport 12: 3859-3863

Chang CC, Lin CJ (2001) Training nu-support vector classifiers: theory and algorithms. Neural Comput 13: 2119-2147

Chatterjee A, Aggarwal V, Ramos A, Acharya S, Thakor NV (2007) A brain-computer interface with vibrotactile biofeedback for haptic information. J Neuroeng Rehabil 4: 40

Daly JJ, Wolpaw JR (2008) Brain-computer interfaces in neurological rehabilitation. Lancet Neurol 7: 1032-1043

Hayashi H, Oppenheimer EA (2003) ALS patients on TPPV: totally locked-in state, neurologic findings and ethical implications. Neurology 61: 135-137

Hill NJ, Lal TN, Schroder M, Hinterberger T, Wilhelm B, Nijboer F, Mochty U, Widman G, Elger C, Scholkopf B, Kubler A, Birbaumer N (2006) Classifying EEG and ECoG signals without subject training for fast BCI implementation: comparison of nonparalyzed and completely paralyzed subjects. IEEE Trans Neural Syst Rehabil Eng 14: 183-186

Hjorth B (1975) An on-line transformation of EEG scalp potentials into orthogonal source derivations. Electroencephalogr Clin Neurophysiol 39: 526-530

Huang D, Lin P, Fei DY, Chen X, Bai O (2009) Decoding human motor activity from EEG single trials for a discrete two-dimensional cursor control. J Neural Eng 6: 46005

Iversen IH, Ghanayim N, Kubler A, Neumann N, Birbaumer N, Kaiser J (2008b) A braincomputer interface tool to assess cognitive functions in completely paralyzed patients with amyotrophic lateral sclerosis. Clin Neurophysiol 119: 2214-2223

Iversen I, Ghanayim N, Kubler A, Neumann N, Birbaumer N, Kaiser J (2008a) Conditional associative learning examined in a paralyzed patient with amyotrophic lateral sclerosis using brain-computer interface technology. Behav Brain Funct 4: 53

Jasper HH, Andrews HL (1938) Electro-encephalography. III. Normal differentiation of occipital and precentral regions in man. Arch Neurol Psychiat 39: 95-115

Joseph AB (1985) Design considerations for the brain-machine interface. Med Hypotheses 17: 191-195

Kayagil TA, Bai O, Henriquez CS, Lin P, Furlani SJ, Vorbach S, Hallett M (2009) A binary method for simple and accurate two-dimensional cursor control from EEG with minimal subject training. J Neuroeng Rehabil 6: 14

Keerthi SS, Lin C (2003) Asymptotic behaviors of support vector machines with Gaussian kernel. Neural Computation 15: 1667-1689

Kennedy PR, Bakay RA, Moore MM, Adams K, Goldwaithe J (2000) Direct control of a computer from the human central nervous system. IEEE Trans Rehabil Eng 8: 198-202

Krusienski D J, Schalk G, McFarland D J and Wolpaw J R (2007) A mu-rhythm matched filter for continuous control of a brain-computer interface IEEE Trans Biomed Eng 54 273-80

Kubler A, Neumann N, Kaiser J, Kotchoubey B, Hinterberger T, Birbaumer NP (2001) Braincomputer communication: self-regulation of slow cortical potentials for verbal communication. Arch Phys Med Rehabil 82: 1533-1539

Kubler A, Kotchoubey B, Hinterberger T, Ghanayim N, Perelmouter J, Schauer M, Fritsch C, Taub E, Birbaumer N (1999) The thought translation device: a neurophysiological approach to communication in total motor paralysis. Exp Brain Res 124: 223-232

Li Q, Doi K (2006) Analysis and minimization of overtraining effect in rule-based classifiers for computer-aided diagnosis. Med Phys 33: 320-328

Lule D, Zickler C, Hacker S, Bruno MA, Demertzi A, Pellas F, Laureys S, Kubler A (2009) Life can be worth living in locked-in syndrome. Prog Brain Res 177: 339-351 
McFarland D J and Wolpaw J R (2003) EEG-based communication and control: speedaccuracy relationships Appl Psychophysiol Biofeedback 28 217-31

Mockford C, Jenkinson C, Fitzpatrick R (2006) A Review: carers, MND and service provision. Amyotroph Lateral Scler 7: 132-141

Muller KR, Mika S, Ratsch G, Tsuda K, Scholkopf B (2001) An introduction to kernel-based learning algorithms. IEEE Trans Neural Netw 12: 181-201

Mutsaarts M, Steenbergen B, Meulenbroek R (2004) A detailed analysis of the planning and execution of prehension movements by three adolescents with spastic hemiparesis due to cerebral palsy. Exp Brain Res 156: 293-304

Naeem M, Brunner C, Leeb R, Graimann B and Pfurtscheller G (2006) Seperability of four-class motor imagery data using independent components analysis J Neural Eng 3 208-16

Neuper C, Scherer R, Reiner M and Pfurtscheller G (2005) Imagery of motor actions: differential effects of kinesthetic and visual-motor mode of imagery in single-trial EEG Brain Res Cogn Brain Res 25 668-77

Neuper C, Pfurtscheller G (2001) Evidence for distinct beta resonance frequencies in human EEG related to specific sensorimotor cortical areas. Clin Neurophysiol 112: 2084-2097

Pfurtscheller G and Solis-Escalante T (2009) Could the beta rebound in the EEG be suitable to realize a "brain switch"? Clin Neurophysiol 120 24-9

Pfurtscheller G, Neuper C, Brunner C, da Silva FL (2005) Beta rebound after different types of motor imagery in man. Neurosci Lett 378: 156-159

Pfurtscheller G, Lopes da Silva FH (1999) Event-related EEG/MEG synchronization and desynchronization: basic principles. Clin Neurophysiol 110: 1842-1857

Pfurtscheller G, Stancak A, Jr., Neuper C (1996) Post-movement beta synchronization. A correlate of an idling motor area? Electroencephalogr Clin Neurophysiol 98: 281-293

Pfurtscheller G (1992) Event-related synchronization (ERS): an electrophysiological correlate of cortical areas at rest. Electroencephalogr Clin Neurophysiol 83: 62-69

Rao S M, Binder J R, Bandettini P A, Hammeke T A, Yetkin F Z, Jesmanowicz A, Lisk L M, Morris G L, Mueller W M, Estkowski L D and et al. (1993) Functional magnetic resonance imaging of complex human movements Neurology 43 2311-8

Salmelin R, Hamalainen M, Kajola M, Hari R (1995) Functional segregation of movementrelated rhythmic activity in the human brain. Neuroimage 2: 237-243

Sellers EW, Donchin E (2006) A P300-based brain-computer interface: initial tests by ALS patients. Clin Neurophysiol 117: 538-548

Sykacek P, Roberts S, Stokes M, Curran E, Gibbs M, Pickup L (2003) Probabilistic methods in BCI research. IEEE Trans Neural Syst Rehabil Eng 11: 192-195

Velliste M, Perel S, Spalding MC, Whitford AS, Schwartz AB (2008) Cortical control of a prosthetic arm for self-feeding. Nature 453: 1098-1101

Williams MT, Donnelly JP, Holmlund T, Battaglia M (2008) ALS: Family caregiver needs and quality of life. Amyotroph Lateral Scler 9: 279-286

Wolpaw JR, McFarland DJ (2004) Control of a two-dimensional movement signal by a noninvasive brain-computer interface in humans. Proc Natl Acad Sci U S A 101: 17849-17854

Wolpaw JR, Birbaumer N, McFarland DJ, Pfurtscheller G, Vaughan TM (2002) Braincomputer interfaces for communication and control. Clin Neurophysiol 113: 767-791

Wolpaw J R, Birbaumer N, Heetderks W J, McFarland D J, Peckham P H, Schalk G, Donchin E, Quatrano L A, Robinson C J and Vaughan T M (2000) Brain-computer interface technology: a review of the first international meeting IEEE Trans Rehabil Eng 8 164-73

Wolpaw JR, McFarland DJ (1994) Multichannel EEG-based brain-computer communication. Electroencephalogr Clin Neurophysiol 90: 444-449 


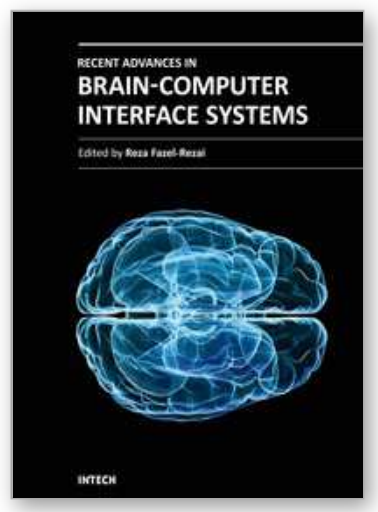

\author{
Recent Advances in Brain-Computer Interface Systems \\ Edited by Prof. Reza Fazel
}

ISBN 978-953-307-175-6

Hard cover, 222 pages

Publisher InTech

Published online 04, February, 2011

Published in print edition February, 2011

Brain Computer Interface $(\mathrm{BCl})$ technology provides a direct electronic interface and can convey messages and commands directly from the human brain to a computer. $\mathrm{BCl}$ technology involves monitoring conscious brain electrical activity via electroencephalogram (EEG) signals and detecting characteristics of EEG patterns via digital signal processing algorithms that the user generates to communicate. It has the potential to enable the physically disabled to perform many activities, thus improving their quality of life and productivity, allowing them more independence and reducing social costs. The challenge with $\mathrm{BCl}$, however, is to extract the relevant patterns from the EEG signals produced by the brain each second. Recently, there has been a great progress in the development of novel paradigms for EEG signal recording, advanced methods for processing them, new applications for $\mathrm{BCl}$ systems and complete software and hardware packages used for $\mathrm{BCl}$ applications. In this book a few recent advances in these areas are discussed.

\title{
How to reference
}

In order to correctly reference this scholarly work, feel free to copy and paste the following:

Dandan Huang, Xuedong Chen, Ding-Yu Fei and Ou Bai (2011). A Two Dimensional Brain-Computer Interface Associated with Human Natural Motor Control, Recent Advances in Brain-Computer Interface Systems, Prof. Reza Fazel (Ed.), ISBN: 978-953-307-175-6, InTech, Available from: http://www.intechopen.com/books/recentadvances-in-brain-computer-interface-systems/a-two-dimensional-brain-computer-interface-associated-withhuman-natural-motor-control

\section{INTECH}

open science | open minds

\section{InTech Europe}

University Campus STeP Ri

Slavka Krautzeka 83/A

51000 Rijeka, Croatia

Phone: +385 (51) 770447

Fax: +385 (51) 686166

www.intechopen.com

\section{InTech China}

Unit 405, Office Block, Hotel Equatorial Shanghai

No.65, Yan An Road (West), Shanghai, 200040, China

中国上海市延安西路65号上海国际贵都大饭店办公楼 405 单元

Phone: +86-21-62489820

Fax: $+86-21-62489821$ 
(C) 2011 The Author(s). Licensee IntechOpen. This chapter is distributed under the terms of the Creative Commons Attribution-NonCommercialShareAlike-3.0 License, which permits use, distribution and reproduction for non-commercial purposes, provided the original is properly cited and derivative works building on this content are distributed under the same license. 